case by the fact, that nutritional supplementation and recovery of normal body weight led to the resolution of free gas, without any other therapeutic intervention. Strict confinement to bed with restriction of activity may exert a beneficial effect, as it may prevent wide fluctuations of intra-alveolar pressure and recurrence of alveolar leaks. The extensive amount and wide distribution of free air in our patient was documented in detail, not only with radiographs, but also with CT of the neck, chest, retroperitoneal space and pelvis, and the resolution of free gas was followed up with serial CT scans.

Relative sinus bradycardia and hypotension in our patient were attributed to autonomic dysfunction which, when observed in such patients, is generally benign. However they have to be treated in order to avoid serious haemodynamic disorders, ${ }^{4}$ especially when electrolyte abnormalities due to malnutrition, vomiting or diuretic and laxative abuse coexist. ${ }^{10}$ In our case there was also a moderate leucopenia obviously due to bone marrow hypoplasia. The decrease of blood cell count

1 McAnarney ER. Physical conditions in adolescense. In: Berkow R, Fletcher AJ, eds, The Merck manual of diagnosis and therapy. Rahway, NJ: Merck \& Co, Inc, 1992; pp 227980.

2 Herzog DB, Copeland PM. Eating disorders. $N$ Engl 7 Med 1985; 313: 295-303.

3 Kaplan AS, Katz M. Eating disorders and connective tissue disease. Etiologic and treatment considerations. Psychosomatics 1992; 33: $105-8$.

4 Fergusson RJ, Shaw TR, Turnbull CM. Spontaneous pneumomediastinum: a complication of anorexia nervosa? Postgrad Med F 1985; 61: 815-7.

5 Satoh K, Kawase Y, Kobayashi T, et al. Anorexia nervosa an association with certain types of soft tissue emphysema. Nippon Kyobu Shikkan Gakkai Zashi (fapan) 1994; 32: 685-8.

6 Schulman A, Fataar S, Van Der Spui JW, et al. Air in unusual places: some causes and ramifications of pneumomediastinum. Clin Radiol 1982; 33: 301-6. may manifest as a pancytopenia, although leucopenia seems to be of greater clinical importance in patients with anorexia nervosa and must be closely monitored as it may result in increased susceptibility to infection. The diminished immunological reaction to infections without fever and leucocytosis may impede early diagnosis and lead to widespread infection in these patients. ${ }^{12}$

In conclusion it must be emphasized that the development of soft tissue emphysema in patients with anorexia nervosa is a benign condition which does not demand surgical intervention. However, because of some potentially life-threatening complications, like unexpected cardiovascular collapse, sudden death, etc, ${ }^{10}$ recognition of the primary disorder, hospitalisation and intensive nutritional rehabilitation is necessary. A complete radiographic investigation of the gastrointestinal tract is indicated in order to exclude a perforation with subsequent mediastinitis or peritonitis. A potential pitfall is that of free gas below the diaphragm that may lead to unnecessary laparotomy. ${ }^{4,7}$

7 Overby KJ, Litt IF. Mediastinal emphysema in an adolescent with anorexia nervosa and self-induced emesis. Pediatrics 1988; 81: $134-6$.

8 Satoh K, Ohkawa M, Tanabe M, Suwaki H. Anorexia nervosa with soft tissue emphysema in multiple locations (letter). AfR 1994; 163: 484.

9 Lands L, Desmond K, Demisio D, Pavilanis A, Coates AL The effects of nutritional status and hyperinflation of respiratory muscle strength in children and young adults. respiratory muscle strength in children
Am Rev Respir Dis 1990; 141: 1506-9.

Am Rev Respir Dis 1990; 141: 1506-9.
10 Sharp CW, Freeman CPL. The medical complications of Sharp CW, Freeman CPL. The medical complications

11 Savvas M, Treasure J, Studdy J, et al. The effect of anorexia nervosa on skin thickness, skin collagen and bone density Br f Obstet Gynaecol 1989; 96: 1392 - 4 .

12 Fukudo S, Tanaka A, Muranaka M, et al. Reversal of severe leukopenia by granulocyte colony-stimulating factor in anorexia nervosa. Am $\mathcal{f}$ Med Sci 1993; 305: 31 - 47 .

\section{Halton General \\ Hospital, Hospital \\ Way, Runcorn, Cheshire WA7 2DA, \\ UK \\ L Martin \\ K Strahan \\ G Murphy \\ Correspondence to: $\mathbf{M r}$ Lee Martin, Royal \\ Liverpool University \\ Hospital, Department of \\ Surgery, 8C Link, Prescot \\ Street, Liverpool L7 8XP, UK}

Accepted 9 December 1996

\title{
Retrieval of intravascular iatrogenic foreign bodies using a nonsurgical technique
}

\author{
Lee Martin, Keith Strahan, Gerry Murphy
}

\section{Summary \\ Two cases are presented in which inter- ventional radiology was used to retrieve foreign bodies from in and around the vascular system.}

Keywords: radiology, foreign bodies, catheters

Vascular access techniques are being increasingly used in the diagnosis and treatment of patients, many of whom are critically ill. As with any invasive technique, complications can occur, one of which is fracture and/or misplacement of guidewires and catheters. In the past, surgical removal of the foreign bodies has been the only option available, however mod- ern interventional radiological techniques can often obviate the need for surgical intervention. We describe two such cases, one involving removal of a knotted guidewire and the other removal of a broken guidewire fragment, both complications occurring during the routine insertion of a central venous catheter.

\section{Case reports}

\section{Case 1}

A central venous catheter was inserted, via a right subclavian approach, for venous access in a 30-year-old woman who had taken a large overdose. Initial entry of the introducer catheter/needle into the vein was difficult, necessitating several passes until good venous flow 
was demonstrated. The spring guidewire was then advanced through the introducer needle. On introduction of the wire, resistance was encountered, which was thought to be secondary to kinking, and the wire was further advanced using a rotational movement to try and overcome this. Further resistance was encountered and on trying to withdraw the guidewire, it was found to be stuck. A subsequent chest $\mathrm{X}$-ray revealed the guidewire had become coiled and knotted behind the medial end of the clavicle with an associated right-sided pneumothorax, which was causing mediastinal shift towards the left (figure 1) and increasing dyspnoea. An intercostal chest drain was inserted, following which the patient became asymptomatic. Percutaneous retrieval of the knotted guidewire was performed using the nonsurgical technique described below. Post-procedure the patient made an uneventful recovery.

Case 2

A central venous catheter was inserted, via a right subclavian approach, in a 52-year-old woman who required parenteral feeding following a laparotomy for resection of a pancreatic carcinoma. Following successful catheter placement in the subclavian vein, resistance was encountered when attempting to remove the spring guidewire. The catheter was withdrawn relative to the guidewire and a further attempt made to remove the wire. Further resistance was again met, and therefore the catheter and guidewire were removed simultaneously. Upon removal it was noted the guidewire was not intact and a chest X-ray revealed the distal fragment to be lodged in the subclavian vein (figure 2). The fragment was removed, again using a percutaneous retrieval recovery.

\section{Technique}

By passing a series of Van Andel Teflon dilators (William Cook Ltd, UK) over the external portion of the guidewire, the percuta-

Figure 1 Chest X-ray showing coiled, knotted guidewire at medial end of clavicle with associated right-sided pneumothorax and mediastinal shift towards the left technique, the patient making an uneventful

neous track was dilated up to $9 \mathrm{~F}$ in case 1 , and a new similar-sized percutaneous track formed in case 2 after a further guidewire had been introduced into the subclavian vein under radiological control, as the guidewire fragment was wholly intravascular. The low resistance of the Teflon enabled relatively easy passage of the catheters. The catheters were then replaced by an $8 \mathrm{~F}$ 'Bright tip' catheter (Cordis Europa, The Netherlands). This catheter has a wide lumen and is braided so that its angled tip can be rotated by rotation of the hub. The 'Bright tip' catheter was fed up to the knot on the guidewire (case 1) or to the fractured guidewire (case 2). Gentle traction on the guidewire enabled the knot to be drawn into the catheter in case 1 , following which the catheter was removed together with the wire as a unit (figure 3). In case 2, a gooseneck snare (Microvena Europa, Germany) was passed through the lumen of the 'Bright tip' catheter in proximity to the foreign body, and the catheter tip rotated, and moved back and forth to snare the floating free end of the broken steel guidewire, following which it was pulled into the sheath. The snare, catheter and guidewire fragment were withdrawn as a unit.

\section{Discussion}

The true incidence of catheter-related embolism is unknown, although it is probably more
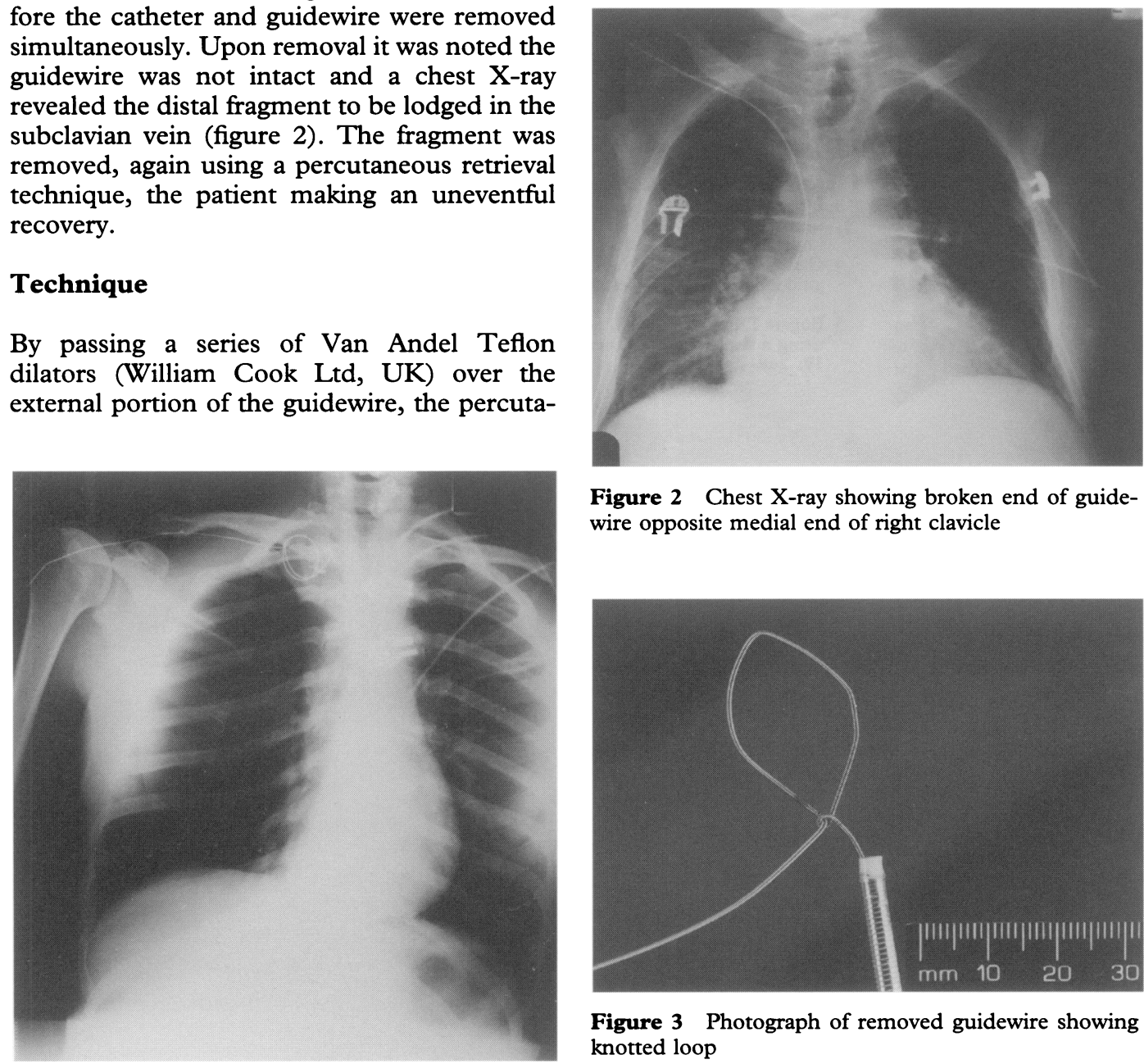

Figure 2 Chest X-ray showing broken end of guidewire opposite medial end of right clavicle

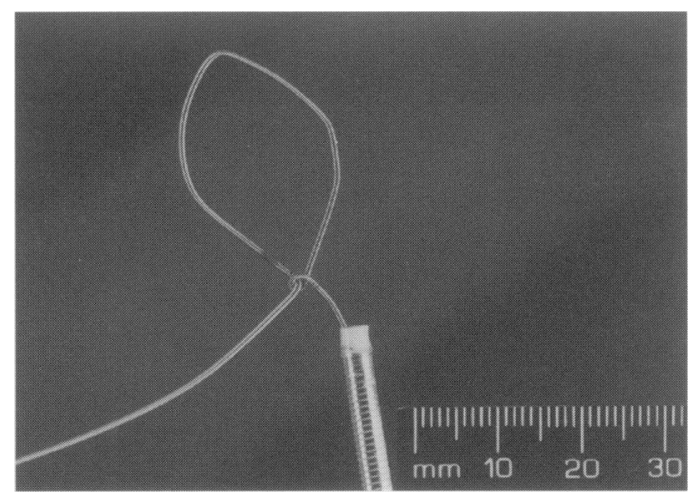

Figure 3 Photograph of removed guidewire showing knotted loop 
common than reported. Interventional radiology can provide an effective means of removing foreign bodies and is becoming increasingly accepted $^{1,2}$. The fact that many patients suffering complications with catheters/wires are seriously ill adds to its attraction. Many devices and techniques have been described. ${ }^{2-6}$ They often involve the use of a wire loop snare, a stone basket of the 'Dormia' type, or grasping forceps. Since the snare was first described by Curry, ${ }^{7}$ it has become the most frequently used technique. ${ }^{8}$ It has the advantages of being simple and safe, the tip being soft and flexible, and readily available in all catheter laboratories. The loop size can be varied greatly and once the foreign body is snared, it gives a good grip. It does, however, have poor torque control, making manipulation difficult and therefore requires dexterity and practice, and the fragment must have a free-floating end to allow it to be snared. Basket catheters are a variant of the snare, having improved torque and hence being easier to manipulate, making them more suitable for removal of short, fragmented, foreign bodies. Again a freefloating end is required and although the instrument is relatively flexible, care must be taken that the rigid tip does not perforate a vascular structure. Bends and curves severely limit the use of rigid/semi-rigid forceps, although newer flexible forceps have been developed. ${ }^{9}$ The major advantage of forceps over snares or baskets is their ability to seize a foreign body at its middle portion, although the

1 Yedlicka JW, Carlson JE, Hunter DW, Castaneda-Zuniga WR, Amplatz K, Nitinol gooseneck snare for removal of foreign bodies; experimental study and clinical evaluation. Radiology 1991; 178: 691-3.

2 Yang F, Ohta I, Chiang H, Lin JC, Shih S, Ma Y. Nonsurgical retrieval of intravascular foreign body: experience of 12 cases. Eur $\mathcal{f}$ Radiol 1994; 18: $1-5$.

3 Fisher RG, Ferreyro R. Evaluation of current techniques for nonsurgical removal of intravascular iatrogenic foreign bodies. AfR 1978; 130: 541-8.

4 Gammill SL, Smith SL. Removal of 'lost' catheters and guide wires without operation. South Med F 1972; 65: 463-

5 Bogart DB, Earnest JB, Miller JT. Foreign body retrieval 19: 248-50. potential for damage to the endothelium and risk of perforation is much greater.

No study exists comparing interventional radiology with conservative treatment and conventional surgery, although Richardson et $a l^{10}$ found that, of 202 cases of catheter emboli described in the literature, $20 \%$ were managed conservatively. In this group, there were no further complications in $56 \%$, non-fatal complications in $21 \%$ and fatal complications in $23 \%$. Mortality rates of up to $40 \%$ have, however, been reported for unremoved emboli, especially in ill patients. ${ }^{10}$ The complications associated with intravascular foreign bodies include arrhythmias, especially with right ventricular emboli, thrombosis, clot embolism, perforation of the blood vessel or cardiac chamber in which the fragment lodges, and sepsis.

Conventional surgery for removal of foreign bodies is associated with a high degree of success, although exposure of the great vessels may involve a thoracotomy or median sternotomy, increasing the risk to the patient, and should now only be considered in those patients in whom interventional radiological techniques have failed, success rates being reported of between $70-100 \% .^{2}$ Therefore, if you have a problem with a broken, knotted or misplaced catheter or guidewire, contact your radiology department, they may be able to help.

We would like to thank Mr J E Pollet for permission to report on his patients.

6 Broomfield DA. Techniques of nonsurgical retrieval of iatrogenic foreign bodies from the heart. Am $\mathcal{F}$ Cardiol 1971; 27: $538-45$.

7 Curry JL. Recovery of detached intravascular catheter or guidewire fragments. A proposed method. $A \Im R$ 1969; 105: guidewire

8 Lybecker H, Anderson C, Hansen MK. Transvenous retrieval of intracardiac catheter fragments. Acta Anaesth Scand 1989; 33: 565-7.

9 Selby JB, Tegtmeyer CT, Bittner GM. Experience with new retrieval forceps for foreign body removal in the vascular, urinary, and biliary systems. Radiology 1990; 176: 535-8.

10 Richardson JD, Grover FL, Trinkle JK. Intravenous catheter emboli: experience with twenty cases and collective review. Am f Surg 1974; 128: 722-7. 